\title{
L'accusation d'émigration des négociants malouins : une justification abusive de la politique terroriste à Saint-Malo
}

Karine Audran

\section{(2) OpenEdition \\ Journals \\ Édition électronique \\ URL : https://journals.openedition.org/ahrf/6863 \\ DOI : 10.4000/ahrf.6863 \\ ISSN : 1952-403X \\ Éditeur : \\ Armand Colin, Société des études robespierristes}

Édition imprimée

Date de publication : 1 septembre 2006

Pagination : 31-53

ISSN : 0003-4436

\section{Référence électronique}

Karine Audran, «L'accusation d'émigration des négociants malouins : une justification abusive de la politique terroriste à Saint-Malo », Annales historiques de la Révolution française [En ligne], 345 | juilletseptembre 2006, mis en ligne le 01 septembre 2009, consulté le 01 juillet 2021. URL : http:// journals.openedition.org/ahrf/6863; DOI : https://doi.org/10.4000/ahrf.6863

Ce document a été généré automatiquement le 1 juillet 2021.

Tous droits réservés 


\title{
L'accusation d'émigration des négociants malouins : une justification abusive de la politique terroriste à Saint-Malo
}

\author{
Karine Audran
}

1789 résonne dans la mémoire collective comme le point de départ de tous les possibles $^{1}$ : en l'espace de quelques semaines, les députés du tiers, bientôt rejoints par des membres de la noblesse et du clergé, s'érigent en Assemblée nationale. La révolution du pouvoir est en marche et la monarchie est impuissante à la contenir, car un acteur déterminant fait son entrée sur la scène politique: le peuple. Les insurrections parisiennes cumulées aux troubles provinciaux transforment le mouvement en expression collective. L'abolition des droits féodaux et l'adoption de la déclaration des droits de l'homme signent l'acte de décès du régime ${ }^{2}$. L'année 1790 , qualifiée d'« année heureuse » par François Furet, engage paradoxalement les députés sur la voie de la radicalisation, lourde de conséquences pour l'avenir. La communion de la nation autour de la fête de la fédération peine à dissimuler les antagonismes naissants. Le rejet des soulèvements populaires, les clivages politiques, ainsi que la question religieuse alimentent un courant d'opposition dont l'émigration est l'expression la plus singulière. Le mouvement de départ en exil des opposants, entamé au lendemain de l'insurrection populaire du 14 Juillet, illustre le rejet du pouvoir en place. Le personnage du noble émigré devient le stéréotype du contre-révolutionnaire ${ }^{3}$. Pourtant, statistiquement les nobles candidats à l'exil sont une minorité.

2 C'est que la question de l'émigration demeure épineuse. Elle recouvre, en effet, sous un terme générique, l'ensemble des opposants au régime, volontaires ou forcés. L'émigration n'est d'ailleurs pas spécifique à la Révolution française, la plus importante étant liée à la révocation de l'Édit de Nantes. Il a toujours existé au cours du XVIII siècle un courant annuel d'émigration, qui incite des familles entières à chercher fortune ou reconnaissance à l'étranger. L'émigration est, pour Roger Dupuy, une des 
conséquences les plus spectaculaires de la Révolution française $e^{4}$. Son originalité tient à sa pluralité : elle est la convergence d'intérêts individuels et collectifs, qui poussent une minorité de la population française à quitter le territoire. Il est d'ailleurs préférable de parler de courants d'émigration, d'origines sociales et géographiques distinctes.

Comment interpréter alors la présence de négociants malouins sur les listes officielles des émigrés ? Serait-ce qu'ils ont été mentionnés absents de leur commune, quand ils n'étaient coupables que d'exercer leur activité ? Mais qu'en est-il des négociants anoblis au cours des XVII et $\mathrm{XVIII}^{\mathrm{e}}$ siècles ? La composition singulière de la société malouine divisée entre un patriciat urbain, vivier des plus anciennes familles du négoce, anoblies pour la plupart sous Louis XIV et une bourgeoisie patriote, composée des talents et d'un négoce, « d'ascension récente et de fortune moyenne ${ }^{5}$ », en déficit de reconnaissance et de pouvoir, transforme le concept d'émigration en un enjeu d'exclusion plus vaste. D'autant que la situation géographique du port en fait un lieu privilégié de débarquement pour une éventuelle reconquête monarchique. Ces négociants ont-ils effectivement migré hors du territoire français ou sont-ils simplement victimes des nombreuses erreurs relevées sur les différentes listes dressées pendant la période révolutionnaire ? Ne sont-ils pas plutôt victimes de leur statut à une époque où le problème des subsistances devient particulièrement aigu?

L'émigration, caractères généraux et législation

4 Avant 1792, le mouvement d'émigration est essentiellement aristocratique, manifestation de refus du nouveau pouvoir en place. Les émigrés disséminés - en Angleterre, en Belgique, le long du Rhin, en Suisse - sont guidés par l'idée d'une fidélité extra-territoriale contrainte par les événements révolutionnaires. L'été 1789 marque la limite entre une émigration « joyeuse », symbolisée notamment par le départ du comte d'Artois le 17 juillet 1789 et une émigration " peureuse » et précipitée au lendemain des journées des 5 et 6 octobre. Le mouvement se renforce l'année suivante avec l'application des décrets sur l'abolition de la féodalité et l'expropriation des biens du clergé. Une loi de décembre 1790 prive de leurs pensions et traitements tous ceux qui persistent à demeurer à l'étranger 6 . 1791 est marquée par l'émigration des militaires, alors que Condé organise l'armée des princes, à Worms. Selon les statistiques, ce sont près de 6000 officiers qui s'exilent, non sans nuire à l'armée révolutionnaire, puis plus tard à la Marine, désorganisée sans ses cadres. Cette émigration spécifique aux officiers nobles de l'armée entraîne de vifs débats à l'Assemblée entre partisans de la Constitution, protecteurs du droit d'émigrer et défenseurs de la Patrie. Une étape est franchie avec la fuite du roi et de sa famille en juin 1791. La législation relativement souple se durcit, d'autant que le comte de Provence est parvenu à rejoindre la frontière. Le décret du 28-29 juin 1791 autorise la sortie du territoire aux seuls étrangers et négociants ${ }^{7}$. Pour éviter d'être assimilés à des émigrés, il faut justifier de la possession d'un passeport, délivré par la municipalité du chef-lieu de district de sa résidence : la surveillance est étroite. L'article quatre de la loi prend en considération le caractère particulier de l'exercice du négoce. Mais qu'est-ce qu'un négociant? « On est négociant en gros, pratiquant la commission, la participation, le change, quelque peu la banque, l'entrepôt et la redistribution des marchandises et le commerce maritime ${ }^{8}$ ». Les aptitudes nécessaires sont diverses mais, pour bien spéculer et s'assurer de confortables bénéfices, il en est une indispensable à un négociant d'envergure nationale: la mobilité. Cette qualité première permet d'entretenir un réseau de relations, dont les intérêts commerciaux, matrimoniaux, sociaux, tissent entre ses membres des liens indissolubles. La confiance, véritable liant, cimente le corps social 
dans un sentiment d'appartenance à l'élite. En 1791, le pouvoir central différencie encore les négociants, en déplacement pour activité, des citoyens s'exilant pour des raisons politiques. Mais ce décret est intéressant à un autre titre, car l'article 6 rappelle que l'or et l'argent sont interdits à l'exportation. Nous touchons là à un point sensible de la législation concernant les émigrés, qui témoigne autant de l'inquiétude pour les autorités de voir se gonfler le complot aristocratique, que de la crainte que ne disparaissent leurs richesses. En août 1791, l'émigration est considérée comme un délit et le terme d'émigré apparaît pour la première fois'. Néanmoins l'Assemblée nationale se sépare le mois suivant sur l'adoption de la Constitution et l'amnistie générale. La loi du 14 septembre 1791 est suspendue : le passeport n'est plus une obligation pour sortir du territoire. En réalité, cette abrogation est directement liée aux intérêts des députés de la Constituante. Ces derniers étant dans l'impossibilité de se présenter aux élections de l'Assemblée législative, en raison du changement des modalités de la loi électorale, s'arrogent ainsi le droit à une émigration légale.

5 Le spectre de la guerre grandissant, les lois se raffermissent, et les textes législatifs se multiplient : treize en 1791, 38 en 1792, 51 dans les neuf premiers mois de l'année 1793, 87 en l'an III. La loi du 8 avril 1792, qui établit les modalités de confiscation des biens est considérée comme la première grande loi contre les émigrés ${ }^{10}$. Dès le mois d'août, des propriétés sont liquidées. L'Assemblée décrète la patrie en danger et interdit la sortie du territoire à toutes les personnes qui ne sont pas munies d'un passeport; les négociants bénéficient encore du précieux sésame, au même titre que les gens de mer, mais cette distinction n'est que temporaire. La suspicion s'installant, les négociants auront bientôt à justifier de leurs déplacements. Le Moniteur du 10 août 1792 dénonce d'ailleurs la facilité coupable avec laquelle sont délivrés les certificats de résidence et autres autorisations.

6 L'année 1792 correspond à un second courant d'émigration, caractérisé par l'exil forcé d'une masse d'individus hétérogènes. Depuis l'élection de l'Assemblée législative le climat politique s'est considérablement dégradé : la déclaration de Pillnitz du 27 août 1791, les propos bellicistes défendus par Brissot, puis bientôt la déclaration de guerre autorisée par le roi, ainsi que les troubles de subsistances au printemps 1792 exacerbent les esprits. Au lendemain de la victoire de Valmy, la Législative proclame la mort de la monarchie constitutionnelle, se soumettant aux sans-culottes parisiens, acteurs du 10 Août et des massacres de septembre. Donald Greer ${ }^{11}$ estime les départs de l'année 1792 deux fois plus nombreux que les exils qui ont eu lieu jusqu'alors. L'exode est alimenté par les autres classes de la société française, les Français de l'Ouest victimes de la guerre civile, les populations déplacées en raison des invasions étrangères - particulièrement les paysans du Bas-Rhin, dont les vagues de départ coïncident avec les avancées de l'armée autrichienne -, enfin les prêtres réfractaires, bannis depuis la loi du 26 août 1792. Pour Massimo Boffa, «les acteurs forcés de cette seconde émigration n'étaient pas des adversaires de la Révolution ${ }^{12}$ ». 1792 représente la ligne de partage du patriotisme: «L'on doit distinguer l'émigration volontaire de l'émigration forcée [...]. En 1791, le parti des aristocrates s'est séparé de la nation de fait et de droit » écrit Mme De Staë ${ }^{13}$. La loi du 28 mars 1793 donne une définition précise du crime d'émigration en temps de guerre, et distingue l'émigration choisie de l'émigration subie. Les émigrés sont déclarés traîtres à la nation, leurs biens sont confisqués et interdiction leur est faite de rentrer en France sous peine de mort. À l'automne, un pas est de nouveau franchi vers l'arbitraire : la loi du 17 septembre 1793 troque le concept mouvant d'émigré pour celui plus encore équivoque de suspect ${ }^{14}$. 
L'ambiguïté du décret vient de ce que, sans nommer formellement la catégorie socioprofessionnelle des négociants, plusieurs des caractéristiques distinctives identifiées leurs sont imputables. L'opacité de la définition d'émigré s'accroît à mesure que le pouvoir insurrectionnel enfle, d'autant que la crise économique aiguise les oppositions sociales. Sous le terme d'« aristocrate", les sans-culottes accusent les bourgeois, et particulièrement les négociants, d'enrichissement ${ }^{15}$. L'hostilité au commerce est un des traits fondamentaux de la mentalité populaire de l'an II $^{16}$. Les négociants et autres marchands sont progressivement absorbés dans le vaste mouvement de suspicion instauré progressivement depuis 1792. Aristocrate, accapareur, émigré : qu'importe désormais le terme. Protégés hier encore dans l'exercice de leurs affaires, les déplacements deviennent douteux, les absences non justifiées trahissent, selon les autorités, des manœuvres subversives. En témoigne l'exemple du fils de Le Breton de Blessin, négociant malouin, inscrit sur les listes d'émigrés de l'an II avec cette observation : " père et mère détenus, ils prétendent que leur fils est en Angleterre pour y a prendre le commerce et la langue ». L'émigration, accusation invoquée jusqu'alors pour des raisons politiques, devient clairement, par l'assimilation des négociants, une suspicion contre le délit d'enrichissement.

$7 \mathrm{Au}$ lendemain du 9 Thermidor, le mouvement d'émigration tend à s'essouffler. Pourtant, après l'échec du débarquement de 4000 émigrés en Bretagne en juin 1795, puis le coup d'État du 18 fructidor an V, l'heure du retour n'a pas encore sonné. L'incohérence marque la législation adoptée après l'an IV. Ainsi le gouvernement donne-t-il, le 30 pluviôse an IV, la possibilité aux émigrés d'obtenir une radiation de leur nom sur les listes. Mais devant la vague incontrôlée des radiations, une loi du 26 fructidor suivant crée «un recueil alphabétique des individus rayés de la liste des émigrés ", imprimé, de manière à freiner l'indulgence avec laquelle les pouvoirs locaux ont jusqu'alors accordé ces suppressions. Pour renforcer le décret, il est encore rappelé, le 17 messidor, qu'une prime de 100 livres sera attribuée à chaque citoyen qui dénoncera, saisira ou arrêtera un émigré. Des restrictions sont apportées en l'an VIII à la définition d'émigré, qui se limite désormais aux seuls combattants des armées royalistes ou étrangères et aux agents du prince. Le temps des suspicions vis-à-vis des négociants semble révolu. L'émigré renoue avec la définition classique du complot aristocratique. Enfin, le 6 floréal an $\mathrm{X}$, un sénatus-consulte proclame une amnistie quasi-générale. "La mode est maintenant de rentrer comme jadis de sortir » note Neuilly dans ses souvenirs ${ }^{17}$.

8 La liste générale des émigrés de toute la République, classée par ordre alphabétique, débutée au printemps 1792 et close par Bonaparte en 1800, comporte 145000 noms, mais est sujette à caution. Certains émigrés y apparaissent en plusieurs occurrences quand d'autres n'y figurent pas. Donald Greer $^{18}$ estime entre 150 et 160 milliers le nombre d'émigrés, représentant $0,6 \%$ de la population française, ce qui ramène le phénomène à de plus justes proportions. Les membres du clergé alimentent ce courant à hauteur de $25 \%$, la noblesse à $17 \%$, les paysans à $19,4 \%$, les ouvriers à $14,3 \%$ et la grande bourgeoisie à $11 \%$. Contrairement aux idées reçues, le mouvement d'émigration est majoritairement le fait d'hommes ou de familles issus du tiers état. Le taux global de l'émigration nobiliaire calculé par Roger Dupuy ${ }^{19}$ à partir de la liste départementale d'Ille-et-Vilaine, donne un pourcentage supérieur de l'ordre de $25 \%$. "Cette représentation qui ne voyait dans l'émigré que l'allié de l'étranger et de l'ennemi de la République allait durer de longues années encore et contribuer à cristalliser en quelque sorte l'image classique qui n'avait jamais été celle que d'une minorité très limitée des 
gens qui avait franchi la frontière depuis $1789^{20}$ ». L'émigration, loin d'être un phénomène lié aux comportements de la seule noblesse, est un courant pluriel alimenté par l'ensemble de la société française, du noble au paysan, en passant par le bourgeois et l'ouvrier. Les raisons en sont diverses: politiques, religieuses, économiques, militaires. Néanmoins, l'essentiel est ailleurs. L'évolution législative témoigne, en effet, de la difficulté qu'éprouvent les autorités à définir exactement l'émigré. Ses caractéristiques évoluent à mesure que le contexte politique se radicalise et les lois votées deviennent draconiennes lorsque la perception du danger s'accroît. L'émigré, figure de la contre-révolution devient dans le courant de l'année 1793, un suspect parmi d'autres. La loi du 17 septembre, loin de dresser les contours d'une définition précise du suspect, décrète l'application d'un texte élastique, véritable brèche ouverte à l'arbitraire. Les négociants, dans un premier temps épargnés, se retrouvent bientôt, sous la pression populaire, suspectés. La chasse est désormais ouverte contre les riches et les égoïstes ${ }^{21}$. L'accusation d'émigration n'est alors plus qu'un prétexte légal à l'incarcération et la spoliation des biens. Dans ces conditions, qu'en est-il réellement de l'accusation d'émigration portée contre certains négociants malouins sous la Terreur? Saint-Malo sous la Révolution

9 Saint-Malo est à la fin du XVIII ${ }^{e}$ siècle un port d'envergure internationale, "produit d'un développement interne spontané22 ${ }^{2}$. Port européen de cabotage, spécialisé dans les trafics médiévaux traditionnels (sel, beurre, vin, toiles), Saint-Malo réussit, grâce au dynamisme de sa bourgeoisie marchande, à prendre part aux nouveaux trafics océaniques. Présente à Terre-Neuve depuis le début du XVI siècle, l'économie morutière est l'apprentissage du trafic triangulaire et de l'accumulation lente. Forts de cette expérience, les Malouins s'engagent résolument sur la voie de la prospérité commerciale à la fin du XVII ${ }^{e}$ siècle. Ils sont tour à tour convoyeurs d'argent pour la royauté, corsaires redoutables et précurseurs des trafics en mer du sud. La fin du règne louis-quatorzien correspond à «l'apogée du rôle capitaliste malouin et de son élite marchande ${ }^{23}$ ». En quelques décennies, le Rocher est devenu une ville de millionnaires. Néanmoins, l'embellie économique est de courte durée. L'interruption de la guerre, signée en 1713, sonne le glas des conditions exceptionnelles d'épanouissement du négoce malouin. Les grands trafics risqués et rentables disparaissent. En 1725, le négoce se reporte sur les échanges traditionnels du cabotage, de la pêche à la morue et du commerce de Cadix. C'est que Saint-Malo la pionnière échoue à s'insérer dans le trafic colonial prometteur des Antilles. L'absence d'un hinterland étendu et dynamique, à l'image de Nantes et Bordeaux, véritables complexes maritimo-portuaires, conjuguée à une certaine évolution sociale du négoce malouin au XVIII ${ }^{e}$ siècle (une bourgeoisie, parfois anoblie, qui tend à devenir passive) explique en partie cette mutation. SaintMalo demeure néanmoins en cette fin de XVIII siècle un port de premier plan ${ }^{24}$, ayant participé depuis deux siècles aux plus grands trafics mondiaux.

La Révolution française et son cortège de bouleversements ne font qu'accélérer un déclin économique déjà prononcé. Pour Roger Dupuy, cette "période [...] est un des accidents majeurs de l'histoire de Saint-Malo ${ }^{25} »$. Elle met un terme à une croissance pluriséculaire. Les Malouins assistent impuissants à l'émiettement de leur influence politique, dès les premiers mois de la Révolution. L'échec de l'adoption d'un département de la Rance est précédé de la sécession du faubourg de Saint-Servan. Rattachés à Saint-Malo depuis 1753, les Servannais, arguant de leur poids démographique et de leur dynamisme économique, érigent une municipalité autonome le 25 décembre 1789. Cette situation est officiellement reconnue par le conseil 
départemental en 1791. En l'espace de quelques mois, le Rocher n'est plus qu'un district, sous contrôle rennais. De fait, Saint-Malo perd également son évêché, celui-ci devant désormais coïncider avec l'administration départementale. Surtout, l'autonomie acquise par Saint-Servan entraîne la perte pour Saint-Malo de la moitié de ses habitants.

11 Les événements révolutionnaires exacerbent des tensions politiques latentes. La société malouine est, en effet, dirigée par une oligarchie, « une ploutocratie noble d'origine négociante ${ }^{26} »$, désavouée dès le déclenchement de la crise révolutionnaire par une bourgeoisie patriote, lassée d'être écartée du pouvoir. "Mais peut-on bien nommer ainsi une assemblée composée d'une trentaine d'armateurs et de capitaines de navires qui s'établissent eux-mêmes les représentants d'une population de 25000 âmes? Les charges municipales sont en quelque sorte héréditaires : cinq ou six familles, qui se croient sans doute patriciennes, sont en possession depuis bien des années de former cette extraordinaire aristocratie ${ }^{27} »$.

Eugène Herpin qualifie cette élite de "gros bourgeois ", assimilant leur pratique du pouvoir à un "fief héréditaire», conséquence d'un «injuste ostracisme ${ }^{28}$ ». L'accaparement du pouvoir par cette minorité semble acquise et, si les termes employés diffèrent selon les auteurs, ils expriment tous cette réalité. Ces « Messieurs de SaintMalo $^{29}$ » forment « un véritable patriciat urbain, combinant les attributs de la richesse et du statut juridique, du prestige et de la puissance, héritages d'une position sociale éminente tenue dans la ville et la province depuis un siècle, voire plus ${ }^{30} »$. Le noyau central de cette élite, constitué d'une douzaine de familles anoblies depuis la fin du règne de Louis XIV, est composé d'une noblesse commerçante hétérogène. Véritables "métis sociaux ${ }^{31}$ ", ces hybrides ne peuvent représenter les intérêts du tiers, dont ils ont abandonné la roture, mais n'appartiennent pas plus à la noblesse, qui les méprise. En conflit ouvert avec une bourgeoisie patriote en pleine formation, les tensions pour le pouvoir sont vives. L'émergence d'une couche moyenne d'armateurs et de négociants d'extraction récente, associée aux professions libérales, juridiques et médicales crée le terreau favorable à l'épanouissement d'une bourgeoisie révolutionnaire ou plébéienne. Ces deux catégories forment un groupe de pression homogène, car nombreux sont les liens qui les unissent. Il n'est ainsi pas rare de trouver dans les armements maritimes des quirataires ou des capitaux issus de la bourgeoisie des talents. La Révolution est l'occasion pour ces « petits bourgeois » d'imposer leur participation à la représentation politique.

13 Sébire l'Ancien maire de la ville et représentant de cette oligarchie urbaine conscient de ces enjeux, invite les représentants des communes et des corporations à se joindre à la municipalité en place. Un conseil permanent est institué en attendant l'élection d'une nouvelle municipalité. Onze conseillers sont élus, et parmi eux quelques négociants-armateurs ou capitaines: Duchesne Saint-Verguet père, Cosson, Danycan, Apuril de Kerloguen, Deshais. Vingt-quatre notables complètent le bureau ${ }^{32}$. Lorsque, selon le décret du 14 décembre 1789, les municipalités sont renouvelées partiellement, Tréhouart de Beaulieu est élu maire, Chifoliau, Leyritz, Jacob, Duguen fils, Brault et Le Roy, officiers municipaux ${ }^{33}$.

14 La bourgeoisie patriote qui prend le pouvoir en 1789, se maintient jusqu'en juillet 1793 . «La complexité sociale du microcosme malouin explique l'inévitable morcellement des choix politiques ${ }^{34} »$. Bastion d'un patriotisme modéré jusqu'en 1793, Saint-Malo n'en demeure pas moins un refuge accueillant pour les aristocrates. Sa situation 
géographique, proche de l'Angleterre, en fait un lieu stratégique pour les défenseurs des princes émigrés, et attire les soupçons des représentants en mission, soupçons d'autant plus prononcés que parlant de la composition de la société malouine Roger Dupuy exprime celle-ci en termes de "concentration aristocratique» et de «densité nobiliaire $\aleph^{35}$. En dépit d'un certain engouement pour les idées de la Révolution, certains patriotes n'hésitent pas à qualifier la ville d'« autre Coblence ». La complexité de la situation politique de Saint-Malo s'exprime dans ce mouvement de balancier qui oscille entre patriotisme et contre-révolution. Bien que la municipalité ait amorcé une démocratisation en 1792 par l'ouverture à la petite bourgeoisie de la boutique et de l'artisanat, et ait exigé en juillet le départ des familles nobles de la ville, Saint-Malo demeure un lieu idéal d'embarquement pour les îles anglo-normandes. Les petits ports environnants accueillent quotidiennement des émigrés toujours plus nombreux. Mais l'aide apportée aux mouvements d'émigration ne s'arrête pas là. À partir de 1793, SaintMalo devient un lieu de transit de la correspondance des princes et assure, par l'intermédiaire de quelques nobles acquis à la cause, les liaisons épistolaires entre les princes, regroupés outre Rhin, et les émigrés basés sur l'île des amis ${ }^{36}$. La conspiration de La Rouërie est un exemple bien connu. Le marquis de La Rouërie, met sur pied en mars 1792 un plan de conjuration approuvé et signé des princes, qui vise à prendre les principales villes de la province: son objectif est de se servir de Saint-Malo pour organiser un débarquement massif d'émigrés, en provenance des îles de Jersey et Guernesey, et entamer une restauration de l'Ancien Régime. Démasqués, les conspirateurs sont arrêtés et envoyés à Paris où ils sont exécutés le 18 咆juin 1794.

L'emprise progressive des contre-pouvoirs de la commune insurrectionnelle, issue du 10 Août, et des principaux clubs parisiens, la crise économique aiguë depuis l'automne 1792, la levée des 300000 volontaires, déclencheur de l'insurrection vendéenne, la crainte de l'invasion étrangère, les troubles fédéralistes, les rumeurs de complots contre-révolutionnaires: tout concourt à détériorer irrémédiablement le climat politique. "Si les circonstances ne peuvent donc ni être totalement niées, ni servir à tout expliquer, elles sont avant tout essentielles dans la naissance et l'entretien de cette peur de l'"Autre" qui aboutit aux mesures présidant à la volonté de mettre celui-ci hors d'état de nuire ${ }^{37}$ ». Mais si la Terreur est officiellement mise à l'ordre du jour le 5 septembre 1793, sous la pression des comités sans-culottes parisiens, cette violence est inhérente à la Révolution. Selon Patrice Gueniffey « la terreur, comme moyen, fait son entrée dans la politique révolutionnaire dès 1789 [...] mais c'est seulement deux ans plus tard, en octobre 1791, que le pouvoir révolutionnaire central, en l'occurrence l'Assemblée législative, se résout à l'employer, et au printemps 1793 qu'elle devient à la fois en s'institutionnalisant, la politique de la Révolution et un système ${ }^{38}$ ". Il définit la Terreur comme « l'emploi de la contrainte et de la violence à des fins politiques et dans le silence des $\operatorname{lois}^{39} »$. Elle est donc inséparable de l'existence ou de la prétendue existence d'un ennemi, justification à son recours. L'ennemi prend d'abord la forme du

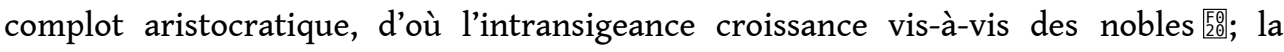
menace de la guerre grandissant, deviennent ennemis tous ceux qui n'adhérent pas ou ne semblent pas adhérer à la République. Dans ce contexte de crainte et de délation, la suspicion est érigée en gouvernement. Le système de la Terreur s'organise alors au local et au national : le Comité de Salut public, crée le 6 avril 1793, composé de douze conventionnels, veille à appliquer la politique défendue par la Convention; le Comité de Sûreté générale, établi à l'été 1792 , supervise la surveillance, les perquisitions, les arrestations, ainsi que l'envoi des accusés au tribunal révolutionnaire. Cet organe 
institutionnel, créé à Paris le 10 mars 1793, remplit une mission déterminante : le jugement des crimes contre-révolutionnaires. Au niveau local, le pouvoir se partage entre les sociétés populaires, les agents nationaux, institués en décembre 1793 et sous l'autorité du Comité de Salut public, les comités révolutionnaires, enfin les représentants en mission. Ces derniers envoyés dans les départements pour veiller à l'application rigoureuse des décisions politiques votées à Paris ont été assimilés par l'historiographie à de véritables proconsuls aux pouvoirs illimités. Après l'envoi de Rochegude, Defermont et Prieur de la Côte-d'Or qui ont la mission d'orchestrer le recrutement des volontaires au printemps 1793, puis le passage à la fin août de JeanBaptiste Carrier, ainsi que Julien et Jeanbon Saint-André à l'automne, c'est au tour du représentant en mission Jean-Baptiste Le Carpentier de faire son entrée à Saint-Malo, le 25 frimaire an II (15 décembre 1793). Originaire du département de la Manche, élu à la Convention en septembre 1792, et proche des Montagnards, il vote la mort du roi en janvier 1793. Envoyé par deux fois en mission dans la Manche et l'Orne, au printemps et à l'été 1793, c'est un homme expérimenté qui est dépêché à Saint-Malo. Soucieux d'appliquer les mesures économiques votées par la Convention, sa réputation est principalement due à sa victoire face aux Vendéens lors du siège de Granville ${ }^{40}$. En raison des tensions politiques locales et de la situation géographique de Saint-Malo, la mission confiée à Le Carpentier par la Convention est de première importance. L'épuration des administrations du district, des sociétés populaires de Saint-Malo et Saint-Servan, ainsi que la lutte acharnée contre toute forme de contre-révolution ont contribué à créer après Thermidor une légende noire de l'action du représentant en mission à Saint-Malo. Le Précis du proconsulat exercé par Lecarpentier dénonce ses actes comme étant ceux d'un bourreau sanguinaire. Mais qu'en est-il effectivement de l'action de Le Carpentier contre les négociants malouins? Peut-on accuser ce dernier de jugements expéditifs à leur encontre?

L'émigration des négociants malouins : un mythe?

16 Les listes alphabétiques des émigrés et condamnés du district de Saint-Malo sont dressées à partir du 7 ventôse an II et durant l'an III $^{41}$. Plusieurs listes ${ }^{42}$ ont été retrouvées et leurs données comparées. Le tableau ci-dessous présente les négociants du corpus ${ }^{43}$ inscrits sur ces états et qualifiés « d'émigrés » ou de « condamnés ». 


\begin{tabular}{|c|c|c|c|}
\hline \multicolumn{2}{|l|}{ Emigrés } & \multicolumn{2}{|l|}{ Condammes } \\
\hline Nom-prénoms & Etat & Nom-prénoms & Etat \\
\hline DUBUAT Jean Georges & Négociant & MAGON De La IANDE & $\begin{array}{l}\text { Ex-noble, } \\
\text { négociant }\end{array}$ \\
\hline $\begin{array}{l}\text { HAUTII. IY Louis Mathurin } \\
\text { Bemard }\end{array}$ & Negociant & $\begin{array}{c}\text { MAGON DE La LANDE } \\
\text { Fils }\end{array}$ & $\begin{array}{l}\text { Ex-noble, } \\
\text { négociant }\end{array}$ \\
\hline IAI.IOBERT Fils aine & Négociant & $\begin{array}{c}\text { MAGON COETIZAC } \\
\text { Fils }\end{array}$ & - \\
\hline JAILOBERT Le jeune & Negociant & $\begin{array}{l}\text { MAGON VILI EHLCHET } \\
\text { Pere }\end{array}$ & $\begin{array}{l}\text { Ex-noble, } \\
\text { négociant }\end{array}$ \\
\hline $\begin{array}{l}\text { MESI E GRANDC IOS Pere } \\
\text { Picre Jacques }\end{array}$ & Négociant & $\begin{array}{c}\text { THOMAZEAL" Pére } \\
\text { Louis Nicolas }\end{array}$ & Marchand \\
\hline $\begin{array}{l}\text { MESL L GRANDCLOS Fils } \\
\text { Stanislas }\end{array}$ & Négociant & & \\
\hline $\begin{array}{c}\text { Fils de Le BRETON } \\
\text { de BIESSIN }\end{array}$ & . & & \\
\hline $\begin{array}{c}\text { Le FER de la SAUDRE } \\
\text { François }\end{array}$ & & & \\
\hline
\end{tabular}

17 Ce travail met en évidence treize acteurs du commerce identifiés, accusés d'émigration sous la Terreur: huit négociants ${ }^{44}$ sont inscrits comme émigrés, cinq comme condamnés pour fait d'émigration. Si l'on compare ce chiffre à l'ensemble des 204 individus exerçant le négoce sur l'ensemble de la période Révolution-Empire, 6,3\% de la profession semble être directement concernée par le phénomène. Si l'on ne tient compte que des négociants condamnés par le tribunal révolutionnaire, le résultat est encore plus significatif: les cinq condamnés, rapportés sur les listes, représentent moins de $2,5 \%$ du groupe ${ }^{45}$.

Le recoupement des sources ${ }^{46}$ nous permet de considérer ces chiffres comme proches de la réalité : la proportion de négociants accusés d'émigration et condamnés est donc marginale. Serait-ce que la menace terroriste s'exerce avec force sur tous, mais ne s'abat que sur quelques-uns, à titre d'exemple ${ }^{47}$ ? Si l'on compare l'impact de la politique terroriste dans les différents ports de France, l'idée générale est celle d'une oppression organisée vis-à-vis du négoce, mais d'un recours sporadique aux condamnations à mort qui font de l'accusation d'émigration proférée contre les négociants une affaire anecdotique autour de quelques cas.

Un bilan global dressé par Eugène Herpin qui concerne l'ensemble de la population de Saint-Malo propose des chiffres vraisemblables ${ }^{48}$ de l'ordre de 400 personnes incarcérées, d'une centaine traduites au tribunal révolutionnaire et d'une quarantaine guillotinées. Notre étude se circonscrivant aux seuls acteurs du négoce fait état d'une douzaine de personnalités directement concernées par une accusation d'émigration ${ }^{49}$. O. Pétré-Grenouilleau présentant les chiffres statistiques de la suspicion légalisée vis-àvis des négociants estime qu'à « Bordeaux, sur 200 négociants arrêtés, 159 sont retenus un certain temps, 85 acquittés, 56 libérés, moyennant le paiement d'une forte amende, et 18 sont condamnés à mort. Dans le département nantais de la Loire-Inférieure, l'un des plus touchés par la justice révolutionnaire, avec 3548 condamnations à mort, il faut chercher attentivement, pour découvrir quatre noms de négociants ou de parents de négociants [...]. À Marseille, 41 négociants en tous genres sont signalés comme étant émigrés en août 1793. Ils sont 144 à la fin de l'année, mais Charles Carrière estime que 
moins de $30 \%$ du négoce est touché ${ }^{50}$ ». L'instauration d'une politique dictatoriale est tangible, mais ses effets sur le négoce ne le sont pas particulièrement. Quelles réalités recouvre précisément l'accusation d'émigration?

Il faut comprendre que ces listes ne sont que la partie émergée de l'iceberg, car la Terreur, instituée pour frapper tous les ennemis de la patrie, s'exerce quotidiennement de manière plus insidieuse. La constitution des listes d'émigrés et l'inscription des négociants ne sont pas l'essentiel. Il ne s'agit finalement que d'un marquage officiel, qui ne vise qu'à différencier publiquement certains individus. L'invention du complot des Magon en est l'archétype ${ }^{51}$. Le Comité de Sûreté générale décrète, le 14 octobre 1793, l'arrestation de Magon de La Balue ${ }^{52}$, ancien fermier général, banquier à Paris, accusé avec l'ensemble de sa famille, c'est-à-dire toutes les branches Magon, Magon de La Lande, Magon de La Villehuchet, Magon de La Blinaye, de "s'être concertés avec les émigrés dans un but contre-révolutionnaire, de leur avoir fourni des fonds et d'avoir favorisé leurs projets liberticides ${ }^{53}$ ». Envoyés à Paris, sept membres du lignage Magon comparaissent devant le tribunal révolutionnaire, le 1er thermidor an II, et sont exécutés le jour même. Charles Erasme Magon de La Lande, négociant et ex-noble, inscrit sur les listes d'émigrés, "condamné révolutionnairement » est guillotiné le 7 thermidor pour le même chef d'accusation. Pourtant une lettre adressée par le commissaire du Directoire exécutif près de l'administration municipale de Port-Malo au commissaire du Directoire exécutif près de l'administration centrale du département d'Ille-et-Vilaine, datée du 28 pluviôse an VII ${ }^{54}$ indique qu'il «a été guillotiné à Paris mais je ne crois pas qu'il ait jamais émigré » : l'émigration ne semble être qu'une accusation arbitraire et fallacieuse exercée à l'encontre de négociants socialement et politiquement trop exposés. Les membres les plus influents sont exécutés, tandis que la majorité du clan est provisoirement emprisonnée ${ }^{55}$.

Il est intéressant de noter à la suite du travail de Gilles Fleury ${ }^{56}$, que l'on reproche sans doute plus à Magon de La Balue ses relations antérieures que ses actions immédiates. L'émigration est en réalité une condamnation contre le crime de "négociantisme ». À une époque où la crise économique fait rage, particulièrement celle des subsistances, l'enrichissement est perçu comme une provocation. Carrier le dénonce en ces termes: "Tous les riches, tous les marchands, sont des contre-révolutionnaires, dénoncez-lesmoi et je ferai rouler leurs têtes sous le rasoir national ${ }^{57}$ ». Cette thèse est directement inspirée de l'action des sans-culottes parisiens, qui s'opposent de plus en plus violemment, la situation économique se dégradant, aux détenteurs du capital commercial. "L'hostilité profonde de la sans-culotterie contre la bourgeoisie commerciale se traduit par une volonté punitive et une exaltation terroriste ${ }^{58}$ ». La réglementation du commerce des blés adoptée dans de nombreux départements français à l'automne 1792 ne suffit pas à calmer les insurrections populaires. L'instauration de la Convention a pour effet de libérer de nouveau le commerce des grains, en décembre 1792, sous le rapport du ministre de l'Intérieur Roland, non sans créer de vifs débats à l'Assemblée. Concomitamment à la crise des subsistances, l'assignat poursuit sa chute vertigineuse ${ }^{59}$. Au début de l'année 1793, les manifestations reprennent, sous l'action de Jacques Roux. La Convention adopte le 4 mai 1793, sous la pression d'une députation du faubourg Saint-Antoine, le maximum des prix des grains. Finalement elle décrète fin juillet 1793 sur les propositions de Collot d'Herbois, une loi contre l'accaparement. La peine de mort est requise contre les accapareurs, c'est-à-dire contre « les commerçants qui ne feraient pas la déclaration de leurs stocks de denrées de première nécessité et n'en afficheraient pas la liste à leur porte ${ }^{60} »$. En réalité la 
rareté des denrées est moins le fait de l'accaparement, que la conséquence d'une insuffisance de production. La guerre aux frontières, la guerre civile en Vendée, la situation économique difficile : tout concourt à la suspicion vis-à-vis des négociants qui sont par nature des spéculateurs. C'est dans ce contexte économique défavorable, conjugué aux particularismes sociaux, politiques, religieux, et stratégiques que représente la cité de Saint-Malo, que Le Carpentier est accusé d'avoir orchestré une spoliation légalisée des négociants malouins.

Le comité de surveillance, réorganisé par le représentant en mission en pluviôse an II, se lance dans une politique d'arrestations et de fouilles, reprochant aux négociants malouins de soutenir les contre-révolutionnaires vendéens: "Vous ne pouvez plus chers Représentants, vous dissimuler que les infâmes Anglais, Nantais, Rennais et Malouins n'aient perpétué cette bande de brigands de la Vendée, et le plus sûr moyen de les détruire est de frapper sans relâche les égoïstes fortunés ${ }^{61}$ ». Le précis du proconsulat ${ }^{62}$ apporte en annexe $n^{\circ} 15$ une lettre du comité de surveillance au représentant Lecarpentier à Granville qui donne le ton des « dons volontaires » :

«Un de nos collègues eut une petite entrevue avant-hier avec le citoyen DupuyFromy, père ; le bonhomme ignorant l'époque de ton retour dans nos murs (c'est le sens de sa lettre), s'empressa de nous faire son offrande civique. Il donna son emprunt volontaire de 15000 L.s.d.

Plus il est prêt à nous compter celle de $60000 \mathrm{~L}=75000$ L.s.d.

Il fait don de 12 quintaux 75 liv. de riz qu'il a fourni à la Municipalité, ainsi que de 38 boisseaux de froment, dont 10 qu'il a fait passer aujourd'hui au grenier de la ville. En outre 1188 liv. de fer provenant des balcons et rampes de maison.

Avec observation qu'il regrette n'en pouvoir pas donner davantage pour le présent, vu ses pertes considérables; assurant qu'il sera dans toutes les occasions disposés à concourir de tous ses moyens à l'affermissement de la République.

Le citoyen Jean Duguen, nous a fourni un tableau de son avoir, qui nous a paru bien embrouillé. Il saisit ton absence pour offrir :

Un contrat sur le-devant Clergé, au principal de 13000 L.s.d.

Deux idem, de l'emprunt de l'ancienne compagnie des Indes, de $1000 \mathrm{~L}$. chaque, 8 000 L.s.d.

5000 L. placées dans l'emprunt volontaire, 5000 L.s.d. $=25000$ L.s.d.

Il offre encore quelques marcs d'argenterie, inutiles à un Républicain. Il nous observe que ses profits sur les Corsaires ne couvrent pas les pertes qu'il a faites tant en Amérique qu'ailleurs. Enfin, son tableau d'infortune est sur le bureau. Nous croyons devoir attendre ton retour pour que tu l'endoctrines, car il paroit dur et il a peur. Bien du plaisir que nous te désirons à la fête. Nous en avons beaucoup à voir l'embarras de nos nouveaux convertis ».

La menace de la Terreur écartée, les langues se délient bientôt pour dénoncer le système. Le témoignage de la citoyenne Dulesnage est intéressant à cet égard. Citée en annexe du Précis du proconsulat de Lecarpentier comme ayant effectué un don civique de $30000 \mathrm{~L}$ en messidor an II, cette dernière réclame deux ans plus tard le remboursement de cette somme injustement versée. Conscient des abus, le ministre des Finances s'adresse ainsi en prairial an IV (mai 1796) à l'administration centrale du département d'Ille-et-Vilaine: «La citoyenne Dufresne Veuve Delesnage, réclame, Citoyens, la restitution d'une somme de $32268 \mathrm{~L}$ en numéraire et argenterie, avec une boîte d'or, qu'elle a été contrainte de donner pendant le règne de la tyrannie pour éviter la mort, dont elle était menacée [...]. Elle observe qu'elle n'offrit tout ce qu'elle possédait au Comité révolutionnaire que dans le désespoir ou l'avaient jeté les menaces de la faire périr, si elle ne prenait pas ce parti ${ }^{63} »$. 

l'an IV ${ }^{64}$ leur donne l'occasion d'adresser à l'administration des requêtes dénonçant ce pillage institutionnalisé. Ainsi, Magon Villehuchet, parent du lignage Magon et dont le père ${ }^{65}$ est inscrit sur la liste des condamnés pour émigration, évoque clairement la situation économique dramatique dans laquelle se trouve sa famille depuis la mission de Le Carpentier à Saint-Malo :

«Je prie d'observer avec attention a qu'il appartiendra que les gens dont les propriétés ont été brulées dès le commencement de la révolution qui ont perdu à cette époque leurs rentes et qui ont été vollés et pillés plusieurs fois dans les différentes visites domiciliaires qui ont été faites chez eux qui ont perdus leurs biens à l'Amérique dont les biens ont été saisis séquestrés et sous la main de la nation pendant long temps, qui ont été détenus et enfermés injustement pendant près d'un an, dont le chef a péri et dont toutes les marchandises et par conséquent toute leur aisance commercialle a été pendant leur détention saisie et vendue au maximum, et le produit du tout versé au District de Morlaix, qui en a encore les fonds en papier, cela est la connaissance de tout le monde, et se trouve totallement perdu : qui ont des charges des dettes, et des rentes viagères à payer qui ont eu bien des pertes par ailleurs et ces gens là dis-je, prient et demandent qu'ont ait quelque égard à leur position et voila notre cas $^{66} \%$.

Au-delà de l'exemplarité, cette lettre énumère succinctement les rouages de la pression terroriste exercée à l'encontre des négociants : multiplication des visites domiciliaires, incarcération, jusqu'à l'exécution dans le cas particulier du lignage Magon.

Cette missive est accompagnée d'une note du même auteur évoquant une nouvelle fois la situation pénible à laquelle il est réduit et qui justifie, selon lui, la suspension du paiement de l'emprunt forcé.

« Port-Malo, 22 nivôse an IV, voila Citoyen et cher compagnon d'infortune une petite notte d'observation dont je vous prie de faire usage au besoin, comme il s'agit de l'impot forcé, et que, le département dont vous êtes, à de l'influence là dedans, je vous serai obligé d'observer que notre famille est une de celle que le reigne de Le Carpentier à le plus maltraitté vous priant d'y avoir quelque égard : notre position n'est plus la même, tout a changé et les fortunes ont passées en d'autres mains $[. . .]^{67} 》$.

Quant à Despecher Fils, il pose sur l'état commercial de la place maritime un regard particulièrement pessimiste :

«L'état de notre commune jadis si riche et dont le commerce étoit si florissent, est aujourd'hui tellement changé que pour ne pas porter atteinte au crédit de nos anciens négociant, nous avons dans la fixation de leur fortune présente consulter plus leur intérêt avenir, que leur intérêt présent; ainsi citoyens administrateurs, nous osons espérer, qu'instruits au motif qui vous présente encore d'assez forts capitaux dans nos anciennes maisons de commerce vous aurez égard aux pertes incalculables qu'elles ont faites pendant le Proconsulat de Carpentier ${ }^{68}$ ».

L'oppression ne réside pas dans les condamnations décrétées par le Tribunal révolutionnaire, dernier stade avant la guillotine, mais dans le climat de terreur et de suspicion créé par le comité de surveillance et dirigé par le représentant en mission, Le Carpentier. L'accusation d'émigration, sans que soit démontré le départ effectif en exil, est une forme de négociantisme déguisé, dans une cité où l'alliance du négoce et de la noblesse crée la confusion. Invités à se racheter, les négociants accusés d'émigration se retrouvent prisonniers d'un système pernicieux de surenchère des dons. Mais cette politique terroriste a-t-elle réellement nui au commerce malouin? Plus précisément, 
l'inscription sur les listes d'émigrés marque-t-elle la fin de la pratique négociante pour ces accusés?

Cette interrogation nous paraît fondamentale pour comprendre l'évolution des négociants incriminés. Une première source concerne les radiations de la liste des émigrés, regroupées en $1 \mathrm{Q} 174^{69}$, première étape dans le rétablissement des droits et de l'honneur :

\begin{tabular}{|c|c|}
\hline BODINIER Frères et Sœurs ? & 14 frimaire an IV \\
\hline COURSON Jean Marie & Ant. 28 vendémiaire an IX \\
\hline JALLOBERT Dominique François & Ant. 28 vendémiaire an IX \\
\hline JALLOBERT Deny Dominique & Ant. 28 vendémiaire an IX \\
\hline LE FER La SAUDRE François? & 6 brumaire an $\mathrm{X}$ \\
\hline MACE Gilles Jean François & 22 nivôse an IV \\
\hline MAGON De La LANDE Fils & 19 fructidor an IX \\
\hline MAGON Charles Auguste & 13 frimaire an $X$ \\
\hline MALO DUBREUIL Pierre? & 2 frimaire an IX \\
\hline MESLE GRANDCLOS Pierre Jacques & Ant. 28 vendémiaire an IX \\
\hline MESLE GRANDCLOS Fils Stanislas & 14 nivose an $X$ \\
\hline
\end{tabular}

Ce tableau énumère l'ensemble des Malouins radiés des listes d'émigrés, ainsi que la date de leur radiation. En italique apparaissent les négociants Malouins du corpus, mais ce tableau met en évidence quelques irrégularités car il répertorie des négociants radiés (Bodinier frères et sœurs), qui ne sont pas reportés sur les listes d'émigrés de l'an II et III.

31 Les négociants sont disculpés au plus tard au 14 nivôse an $\mathrm{X}$ et sont publiquement rétablis dans leur intégrité. Les frères Jallobert, Dominique François et Denis Dominique, ainsi que Meslé Grandclos Pierre Jacques et Meslé Grandclos Stanislas Fils, sont des cas pertinents. Accusés d'émigration sous la Terreur, la date précise de leur réhabilitation n'est pas connue, mais celle-ci est effective dans les années 1800-1802. Une lettre manuscrite des frères Jallobert, datée de l'an VI (1797-1798), récuse l'accusation d'émigration. Ils «[...] séparent de nouveau leur cause de celle des émigrés et déclarent qu'ils ne doivent point être rangés dans la classe de ceux qu'atteint la loi du 19 fructidor ». Mais le plus intéressant nous semble être la présence sur la liste des radiations de deux membres du lignage Magon, Magon de La Lande Fils et Magon Charles Auguste ${ }^{70}$. Trois membres du lignage sont pourtant inscrits comme émigrés condamnés, ce qui signifie que l'accusation d'émigration, transformée par le tribunal révolutionnaire en délit, n'a pas entraîné l'application systématique de la peine de 
mort : le clan Magon n'a donc pas été physiquement anéanti. Le recours à la peine de mort a valeur d'exemple: la brutalité et l'apparente incertitude des jugements, la rareté de la condamnation et son exceptionnalité garantissent l'assujettissement de la totalité du clan.

Pourtant dans les faits l'accusation d'émigration n'empêche nullement la reprise des affaires. Ainsi l'exemple des frères Jallobert radiés en l'an IX, dont les parents ont été eux-mêmes détenus, est significatif de l'évolution d'une partie des négociants malouins après la Terreur. Mentionnés dans les actes de l'Amirauté, du Consulat et du Tribunal de commerce, la présence de Jallobert père dans l'armement maritime est formellement attestée dès l'année 1785. Un des fils est progressivement associé aux affaires paternelles entre 1785 et 1792. Pas moins de 22 armements sont enregistrés à Saint-Malo sous l'identité Jallobert-Jallobert fils, principalement à destination de TerreNeuve. La première mention d'un armement sous l'association des frères date de l'an $\mathrm{V}$, ce qui confirme, s'il était besoin, que la pratique du commerce ne souffre pas de l'accusation passée d'émigration. Les affaires reprennent, alors même que la radiation n'est pas encore officielle. L'oppression terroriste n'empêche nullement la passation de pouvoir économique au sein de la famille Jallobert. Une requête manuscrite adressée à l'administration centrale du département d'Ille-et-Vilaine, datée du 10 ventôse an VI (28 février 1798), à l'effet d'obtenir l'autorisation de " continuer leur commerce avec sécurité » donne l'occasion aux frères Jallobert de détailler leurs affaires commerciales: "Les exposants prient l'administration de considérer qu'ils sont commerçants de profession, qu'en ce moment ils ont à la connaissance de tout le commerce plusieurs affaires maritimes et autres en pleine activité, qu'ils ont dans la commune de Saint-Servan un nouvel établissement de fabrique de bière [...]» L'accusation d'émigration les place dans une situation délicate :

«[Elle] leur fait craindre en ce moment que par une fausse interprétation il ne les mette pas à l'abris d'un séquestre [...]. Si un séquestre avait lieu sur le peu de propriétés qui leur restent, il en résulteraient pour eux les plus funestes conséquences, qui seraient en pure perte pour le bien de la chose publique; que cela anéantirait leur crédit, la confiance que l'on a en eux, obligerait leur associé à désarmer leurs navires et à les passer en rente, les obligerait eux-mêmes à fermer leurs magasins et leur brasserie ; que même le doute tient en ce moment leur crédit en échec et arrête l'extension qu'ils pourraient donner à leurs opérations, et va s'il en est prolongé, ruiner leur crédit en France et à l'Etranger, et le mettre hors d'état de remplir les obligations que la situation de leurs affaires les a mis dans la nécessité de contracter. En conséquence ils supplient l'administration de vouloir bien [...] leur délivrer un passeport pour Paris, afin qu'un des deux puisse s'y rendre, à l'effet de poursuivre le définitif de leur radiation [...] ».

Cet acte détaille minutieusement les conséquences qu'implique une inscription sur la liste des émigrés. Réaffirmés dans leur honneur, ils poursuivent plus sereinement, en dépit de la conjoncture, leurs différentes spéculations et suscitent de nouveau la confiance de leur milieu: l'armement de navires corsaires à partir de l'année 1807 atteste de ce crédit retrouvé. Cet investissement hautement risqué ne peut être mené à bien sans l'existence d'un solide réseau financier local et national. Enfin, pour se convaincre de la crédibilité financière et sociale des frères Jallobert après la Terreur, la consultation des sources politiques est un bon indicateur de l'honorabilité potentielle acquise. Présents sur les listes des plus imposés de la commune de Saint-Malo et du département, ils exercent régulièrement les fonctions de juges et suppléants au Tribunal de commerce de Saint-Malo. Présents sur la liste des 600 plus imposés du 
département, la fratrie Jallobert occupe régulièrement des fonctions politiques sous le Directoire, le Consulat et l'Empire. En tout état de cause, la faillite de la brasserie de Jallobert Jeune en avril 1814, ne peut être imputée à l'action menée par Le Carpentier.

Ces quelques sources témoignent du décalage entre le climat de Terreur et l'oppression réellement subie par le négoce. À partir de quelques mesures coercitives contre le monde négociant, l'historiographie du XIX ${ }^{e}$ siècle a forgé un mythe, celui d'une catégorie socio-professionnelle persécutée par le régime, mais il n'est que de suivre la famille Jallobert pour lui faire un sort. L'inscription sur la liste des émigrés ne les empêche nullement de reprendre, au lendemain du 9 Thermidor, le cours de leurs spéculations et ne semble pas nuire non plus à leur longévité dans le monde des affaires. Les Jallobert illustrent l'obsession de toutes les familles négociantes malouines de la période révolutionnaire : la survie économique et politique.

La double originalité de Saint-Malo, géographique et sociale, dans un contexte d'insurrections populaires et de tensions royalistes fortes, en fait naturellement un port suspect. L'arrivé du représentant en mission, Le Carpentier, en décembre 1793 et la réorganisation du comité de surveillance, n'est pourtant pas synonyme d'une traque effrénée aux «riches» et aux «égoïstes». L'accusation d'émigration et la condamnation demeurent rares. Les Montagnards, conscients de la nécessité de l'exemple, y ont épisodiquement recours afin de susciter dans le groupe visé, l'irrésistible envie de prouver son attachement au régime par quelques substantiels dons volontaires.

L'accusation d'émigration ne serait-elle pas finalement qu'un synonyme du terme de suspect, utilisé localement par des autorités conscientes de la situation singulière qu'occupe la ville de Saint-Malo, tiraillée tour à tour entre un patriotisme modéré et un royalisme au service de la reconquête monarchique?

Définir précisément l'émigration, à partir de l'exemple des négociants malouins, est une tâche ardue, mais la mise au jour des rouages de l'application locale de la politique terroriste est riche d'enseignements : l'émigration est un concept mutant qui profite de l'évolution politique pour s'étendre. L'accusation, délibérément ambiguë, légitime le recours à la violence et à l'arbitraire. La peur, entretenue par quelques exemples frappants de condamnation à mort, en réalité statistiquement faibles, fait le reste : les bases du système terroriste sont jetées. Le négociant l'entretient bien malgré lui, en se soumettant aux volontés du comité, en devançant même parfois ses désirs. Pourtant, les humiliations, l'oppression, la mise sous séquestre des biens et des personnes, le pillage systématique, n'anéantissent pas le négoce malouin. Les spéculations, qui n'ont jamais totalement cessé sous la Terreur, reprennent à la chute de Robespierre. Les négociants écartés du pouvoir par le représentant en mission retrouvent leur position économique, sociale et politique. Le négoce malouin reprend le cours de ses affaires, mû par une seule obsession, la survie économique. La violence terroriste ne s'est pas exercée plus implacablement à Saint-Malo qu'ailleurs. Les statistiques tendraient même à prouver que le négoce y a été relativement épargné. "Le négociant martyr » est un mythe forgé par l'historiographie locale du XIX ${ }^{e}$ siècle pour disculper le négoce malouin de sa responsabilité partielle dans la relégation du port à une simple entité régionale. 


\section{NOTES}

1. «L'année sans pareille », selon l'expression de Louis-Sébastien Mercier.

2.Keith M. BAKER parle d'un « processus dynamique de transformation profonde et irréversible dans la société »; cité par Jean-Pierre JESSENNE, Révolution et Empire, 1783-1815, Paris, Hachette, 1993, p. 66.

3.Roger DuPuY, «Émigration/Émigré », Albert SoBoul, Dictionnaire de la Révolution française, Paris, PUF, 1989, p. 413.

4.Ibid.

5.André LESPAGNOL, « Négociants et Ancien Régime en Bretagne à la fin du XVIII ${ }^{e}$ siècle : Le cas malouin ", La Bretagne, une province à l'aube de la Révolution, actes du colloque de Brest, Brest, 1989, p. 33.

6.Roger DupuY, op. cit., p. 411.

7.«Art. 1er. La libre sortie du royaume ne sera permise, jusqu'à ce qu'il en ait été autrement ordonné, qu'aux étrangers et aux négocians français avec les précautions qui vont être indiquées pour les uns et pour les autres [...] Art. 4. Les négocians français et courriers envoyés par lesdits négocians, qui voudront sortir du royaume, seront également munis d'un passeport de la municipalité du chef-lieu du district [...].» «Art. 6. La sortie des armes, munitions, chevaux (autres que ceux qui servent aux courriers, aux étrangers, aux négocians et conducteurs de voitures), et celle des matières et espèces d'or et d'argent, notamment par tous les ports de mer, restent également prohibées jusqu'à nouvel ordre ", Jean-Baptiste DUVERGIER, Collection complète des lois, décrets, ordonnances, règlements et avis du conseil d'État de 1788 à 1824, Paris, 1824.

8.Jean MEYER, L'armement nantais dans la seconde moitié du XVIII' siècle, Paris, 1969, p. 101. 9.Roger DuPuY, op. cit., p. 411.

10. «'Assemblée nationale, considérant qu'il importe de déterminer promptement de la manière dont les biens des émigrés, qu'elle a mis sous la main de la nation par son décret du 9 février dernier, seront administrés, de régler les moyens d'exécution de cette main-mise, et les exceptions que la justice ou l'humanité prescrivent [...] ». JeanBaptiste DUVERGIER, op. cit.

11.Donald GREER, The Incidence of the Terror during the French Révolution : A statistical interpretation, Harvard University Press, 1935, cité par Jean VIDALENC, Les Émigrés Français, 1789-1825, Caen, 1963, $471 \mathrm{p}$.

12.Massimo BOFFA, « émigrés », François FuRET et Mona Ozouf (dir.), Dictionnaire critique de la Révolution, Paris, 1992, p. 346-359.

13.Ibid.

14.Sont déclarés suspects, selon la loi du 17 septembre 1793, rapportée par Merlin de Douai : 1-Ceux qui, soit par leur conduite, soit par leurs relations, soit par leurs propos, ou leurs écrits, se sont montrés partisans de la tyrannie ou du fédéralisme et ennemis de la liberté. 2-Ceux qui ne pourront pas justifier, de la manière prescrite par la loi du 21 mars dernier, de leurs moyens d'exister et de l'acquit de leurs devoirs civiques. 3Ceux à qui il a été refusé des certificats. 4-Les fonctionnaires publics suspendus ou destitués de leurs fonctions par la Convention nationale ou par ses commissions, et non réintégrés, notamment ceux qui ont été ou doivent être destitués en vertu de la loi du 14 août dernier. 5-Ceux des ci-devant nobles, ensemble les maris, les femmes, les frères, mères, fils ou filles, frères ou sœurs et agents d'émigrés, qui n'ont pas constamment 
manifesté leur attachement à la Révolution. 6-Ceux qui ont émigré dans l'intervalle du 1er juillet 1789 à la publication de la loi du 8 avril 1792, quoiqu'ils soient rentrés en France dans le délai fixé par cette loi, ou précédemment; Louis JАСОВ, Les suspects pendant la Révolution (1793-1792), Paris, 1952, p. 55.

15.« Les aristocrates, ce sont tous les riches, tous les gros marchands, tous les accapareurs, les saute-ruisseaux, les banquiers, les cours-tôt de boutique, tous les gens de la chicane et tous ceux qui ont quelque chose ». Un orateur populaire de la section du Mail, le 21 mai 1791, cité par Albert SoBoul, Les Sans-culottes parisiens en l'an II. Mouvement populaire et gouvernement révolutionnaire (1793-1794), Paris, Seuil, 1968, p. 26. 16.Ibid.

17.Massimo BoffA., op. cit., p. 350.

18.Donald Greer, cité par Massimo Boffa (ibid., p. 347).

19.Roger DupuY, «Capitation nobiliaire et émigration en Ille-et-Vilaine (1789-1793) », Noblesse française-noblesse hongroise, XVI ${ }^{e}-X I X^{e}$ siècle, Paris-Budapest, 1981, p. 93-98.

20.Jean VIDALENC, op. cit., p. 429.

21." Riches égoïstes, c'est vous qui causez tous nos maux ! C'est dans vos maisons, c'est dans vos conciliabules ténébreux que les trahisons et la ruine se préparent ; c'est avec votre or que la corruption produit le désordre et les sanglants revers de nos armées. Vous demandez quels sont les reproches que l'on peut vous faire ? La misère de vos concitoyens, les souffrances longues et continuelles des familles honorables de ceux qui donnent leur sang pour la défense de vos propriété, ne déposent-elles pas contre vous? Leurs larmes, leur désespoir, qui en a tari la source, ne doivent-ils pas être attribués à votre odieuse cupidité ? [...] C'est donc avec raison que la défiance s'étend aujourd'hui sur tous ceux qui veulent de la fortune et du pouvoir. Ce sont de faux patriotes, de vrais contre-révolutionnaires, qui n'attendent que le moment de se vendre au premier tyran qui voudra les payer. La République ne peut s'établir qu'en les rejetant de son sein ; il ne doit entrer dans sa composition que des éléments purs. Dans ses premiers moments de fermentation, elle a déjà vomi une partie du limon, le clergé et la noblesse ; il lui reste encore à se purger des égoïstes, des lâches, des traîtres et des fripons [...] » Louis JACOB, op. cit., p. 71-72.

22.André LESPAGNOL, " Saint-Malo, port mondial (XVI-XVIII ${ }^{\mathrm{e}}$ siècles) ", André LeSPAGNOL (dir.), Histoire de Saint-Malo et du pays malouin, Toulouse, 1984, p. 89-137.

23.Ibid.

24.À la veille de la Révolution française, Saint-Malo est au cinquième rang français en nombre d'armements, au troisième rang en matière de construction navale.

25.Roger DuPuY, « Un monde qui s'estompe (1789-1848) », André LESPAGNOL (dir.), Histoire de Saint-Malo et du pays malouin, Toulouse, 1984, p. 215-234.

26.André LESPAGNOL, op. cit., 1989, p. 34.

27.Extrait du cahier de la juridiction de Saint-Malo, Roger DupuY, op. cit., p. 216.

28.Eugène HERPIN, Saint-Malo sous la Révolution 1789-1800, Rennes, 1931, 1e partie.

29.Pour reprendre le titre de la thèse d'André LESPAGNOL.

30.André LeSPAGNOL, op. cit., 1989, p. 33.

31.Jean MEYER, La noblesse bretonne au XVIII siècle, Paris, 1966, chapitre XIV et XV, cité par André LESPAGNOL, op. cit., 1989, p. 34.

32.Dont : Marestier, Halot-La Fontaine, Canneva, Dupuy-Fromy, Grandclos Meslé, Fournier-Dumanoir, Harrington, Guillemaut-Despecher, Chenu Pied-Noir, Dessaudrais Sébire, Le Breton de La Vieuville, Le Fer Beauvais, Marchand et Foucher. 
33.Tillard de La Hurie, Bossinot de Vauvert, Régnault, Potier de La Formandière, Louvel père, Rays, Martin, Chassin de Kergommaux, Sébire Aîné, Jacotard, Jallobert fils, Treffegain, Faucher et Bataille, font partie des notables.

34.Roger DuPUY, op. cit., p. 219.

35.Ibid.

36.L'île de Jersey.

37. Michel BIARD, Pascal DUPUY, La Révolution française. Dynamiques, influences, débats

(1787-1804), Paris, A. Colin, 2004, p. 96.

38.Patrice GUENIFFEY, La politique de la Terreur, Essai sur la violence révolutionnaire, 1789-1794, Paris, Fayard, 2000, 376 p.

39.Ibid.

40.Yves-Marie LE TROQUeR, Politisation et acculturation républicaine dans le district de SaintMalo (1792-an V), mémoire de maîtrise dirigé par S. BIANCHI, Rennes II, 2003, p. 57-58.

41.AD Ille-et-Vilaine, sous-série $1 Q$.

42.AD Ille-et-Vilaine, 1Q 161 listes des émigrés du district de Saint-Malo, an III ; 1 Q 162 listes des émigrés du district de Saint-Malo an II ; 1Q 166, liste des émigrés du département d'Ille-et-Vilaine, an IV-an VI ; 1Q 164, liste des condamnés, district de Saint-Malo.

43.Le corpus des négociants malouins représente 153 négociants ou familles négociantes, soit 204 individus recensés dans les minutes du Tribunal de Commerce de Saint-Malo, les rôles d'armements, les investissements fonciers, ainsi que les listes d'électeurs.

44.Deux autres négociants ont été identifiés : Louis Isaïe Desegray, dit Ledet, négociant et Ollivier Septan, commis négociant. Ces deux acteurs du commerce n'ont pas été retenus dans notre corpus en raison du déficit de données économiques, politiques et sociales les concernant.

45.Seul, Magon de la Lande est guillotiné à Paris le 7 thermidor an II. Concernant Meslé de Grandclos, Eugène Herpin mentionne - dans Saint-Malo sous la Révolution, p. 184, n. 547 - son arrestation le 9 décembre 1793, ainsi que sa condamnation et sa décapitation à Paris le 27 juillet 1794. Mais cette version est erronée. Alain ROMAN - Meslé de Grandclos (1728-1806), mémoire de DEA, Rennes II, 1993, p.14 - rappelle en effet que l'armateur parvient à s'enfuir dès le 10 décembre 1793, vraisemblablement aidé en cela par ses geôliers, et gagne l'Angleterre, via Guernesey. Il ne peut donc avoir été guillotiné au mois de juillet suivant. Il décède à Villiers-Bocage, dans son lit, le 30 janvier 1806 à l'âge de 78 ans. Selon René Kerviller - Les Filiations bretonnes - son fils, Stanislas Meslé de Grandclos, décède en 1844. Quant à Thomazeau Père, une lettre manuscrite retrouvée dans le fonds des Archives municipales de Saint-Malo, 32W 283, témoigne de sa détention à la maison d'arrêt de Saint-Servan en 1800 (il réclame son transfert à l'hospice de Saint-Malo, pour raison de santé. Le transfert lui est accordé en pluviôse an VIII - janvier 1800 - par le Directeur du jury de l'arrondissement de Saint-Servan). En revanche, nous ignorons les conséquences précises de la condamnation de Magon de La Lande et de Magon de La Villehuchet père.

46.AD Ille-et-Vilaine, sous-série $1 Q$, listes des émigrés du district de Saint-Malo, du département de l'Ille-et-Vilaine; AM de Saint-Malo, 32W 282, émigrés, liste du département et du district, Saint-Servan, LL 106, Liste d'émigrés, Saint-Malo. 47.Patrice GUENIFFEY, op. cit., p. 32 : «L'asservissement de tous exige que chacun vive dans une crainte perpétuelle provoquée et entretenue par des exemples éclatants, suffisamment arbitraires et répétés : tous alors prendront peur et comprendront que la 
mort qui a frappé tel coupable pour des motifs incertains pourra, demain, les frapper à leur retour sans plus de formalités ».

48.Roger DuPuY, op. cit., p. 226.

49.Par extension, l'arrestation d'une partie des membres de la famille de ces négociants - parents, épouses, enfants - grossit les rangs des victimes malouines. Mais les données sont lacunaires, qui empêchent une statistique précise. Néanmoins l'imprimé des détenues dans la maison d'arrêt de Port-Malo à l'époque de la fin germinal an II archives municipales, Saint-Malo, LL108 - recense sept femmes proches des négociants accusés : Anne-Hélène Gardin, épouse Magon Coetizac, Marie Meslé Harrington, fille de l'armateur Meslé Grandclos et sœur de Stanislas Meslé Grandclos, Léocardie Marie Harrington, Vve Le Fer, Marie et Marie Hélène le Breton de Blessin, Anne et SainteMarie Jallobert, sœurs des négociants du même nom.

50.0livier PÉTRÉ-GRENOUILLEAU, Les négoces maritimes français, XVII $-X X^{e}$ siècle, Paris, 1997, p. 159.

51.Ernest DAUDET, « La conspiration Magon, récit des temps révolutionnaire », Revue des Deux Mondes, 1911, p. 356-390, p. 624-655.

52.En dépit de leur stature nationale ou plus précisément en raison de celle-ci, Magon de la Balue et son frère Magon de la Blinais sont inconnus des listes départementales. Proches du pouvoir monarchique, ils n'appartiennent pas au monde du négoce malouin local.

53.Roger DuPUY, op. cit., p. 223.

54.AD Ille-et-Vilaine, AQ 188, certificats individuels de non-inscription et de radiation sur la liste des émigrés, département d'Ille-et-Vilaine ; l'acte est en réalité une demande complémentaire de renseignements sur Magon de la Lande Fils.

55.Pour preuve du non-anéantissement du clan Magon, la requête de Magon Coetizac déposée en l'an $\mathrm{V}$ en vue de récupérer ses biens, ainsi que celles de Magon de la Blinaye et de Bodinier - ce dernier est absent des listes officielles d'émigrés, mais semble selon toute vraisemblance sous le coup de cette accusation - AM Saint-Malo, LL102, lettre réclamant la restitution de l'argenterie saisie chez les citoyens Magon la Blinaye, Magon Coetizac et Bodinier, 21 nivôse an V : « Copie de lettre écrite 21 nivôse an V année républicaine par le ministre des Finances aux administrateurs du département d'ille-et-Vilaine. En messidor an II, Citoyens, le Comité de sûreté générale envoya à Port-Malo les citoyens Seneaux et Coulouhon. Ces commissaires qui saisirent dans le même tems chez les citoyens Magon la Blinaye, Magon Coetizac et Bodinier, renferment dans un tonneau tout le numéraire et l'argenterie qu'ils avaient enlevés et l'adressent au Comité de sûreté générale. Il parvint ensuite au magasin des dépouilles des églises où l'argenterie est restée et le numéraire a été versé à la trésorerie nationale sous le nom de Magon de la Blinaye. Comme le citoyen Magon Coetizac réclame seul dans ce moment la restitution de ce dont il a été dépouillé je désirerais que vous puissiez me procurer les procès-verbaux des saisies faites chez les citoyens Magon la Blinaye et Bodinier ou au moins la déclaration de leur prétention. Ces renseignements sont nécessaires pour m'assurer que la nation a reçu la totalité des sommes et argenterie qui sont et peuvent être réclamées. Ils me mettront à portée de statuer sur la demande du citoyen Magon Coetizac [...]».

56.Gilles FLEURY, « Analyse informatique du statut socioculturel des 1578 personnes déclarées suspectes à Rouen en l'an II », Autour des mentalités et des pratiques politiques sous la Révolution française, 112e congrès national des sociétés savantes, Lyon, 1987, p. 9-23. 
57. Olivier PÉTRÉ-GRENOUILLEAU, op. cit., p. 159.

58.Albert SOBOUL, op. cit., p. 35.

59.Florin AfTALION, L'économie de la Révolution française, Paris, 1996, p. l'année 1793, sa valeur réelle correspond à la moitié de sa valeur nominale.

60. Raymonde MONNIER, « Accaparement/accapareurs ", Albert SoBoul (dir.), Dictionnaire Historique de la Révolution française, op. cit., p. 4-5.

61.Roger DuPuY, op. cit., p. 223.

62.Bibliothèque de l'Institut d'Histoire de la Révolution Française (microfiche), François DUAULT, Précis du proconsulat exercé par Lecarpentier sous la tyrannie de Robespierre dans la commune du Port-Malo, Sur des pièces authentiques et sur les témoignages des Malouins, et adressé par eux à la Convention Nationale, 1er germinal an III, Port-Malo, imprimerie de L.H.Hovius, in- $8^{\circ}, 45 \mathrm{p}$.

63.AM Saint-Malo, LL 102, lettres réclamant restitutions de biens.

64.Le 15 frimaire an IV, le Directoire décide la levée d'un impôt de 600 millions sur le quart le plus riche des contribuables, pour rééquilibrer la balance budgétaire, asphyxiée par la dépréciation de l'assignat. François HINCKER, « Emprunts », Albert Soвоul (dir.), Dictionnaire Historique de la Révolution française, op. cit., p. 413-414.

65.Magon Villehuchet Père est indiqué condamné dans les listes des émigrés. Les filiations bretonnes de René KERVILLER mentionnent le décès d'un Messire NicolasFrançois M., Sgr. de la VilleHuchet né à Saint-Malo le 24 octobre 1727, décédé le 20 juin 1794, ce qui correspondrait chronologiquement à l'époque de la condamnation de Magon de la Balue.

66.AD Ille-et-Vilaine, L 668, Emprunt forcé de l'an IV.

67.Ibid.

68.Ibid.

69.AD Ille-et-Vilaine, $1 \mathrm{Q} 174$.

70.L'identification de Magon Charles Auguste est problématique car le nom n'a pas été reporté intégralement sur la liste des radiations, Rennes, AD Ille-et-Vilaine, $1 \mathrm{Q} 174$.

\section{RÉSUMÉS}

Comment interpréter la présence de négociants malouins sur les listes d'émigrés du département d'Ille-et-Vilaine ? L'émigration, formée par un courant hétérogène de candidats à l'exil, est un concept complexe. Le personnage du noble émigré, stéréotype du contre-révolutionnaire est une image créée par l'historiographie. L'essentiel du mouvement est alimenté par des individus ou des familles issus du tiers état. La société malouine dirigée de longue date par une «ploutocratie noble d'origine négociante ", en butte aux velléités de pouvoir d'une «bourgeoisie plébéienne ", devient sous la Révolution l'enjeu d'un patriotisme acharné autant que d'une menaçante reconquête monarchique. Dans ces conditions particulières, quelles réalités confère précisément l'accusation d'émigration portée contre quelques négociants malouins, dont certains ont été anoblis entre la fin du XVII ${ }^{\mathrm{e}}$ et le début du XVIII ${ }^{\mathrm{e}}$ siècle? 
The Accusation of Emigration among Malouin Merchants: a Misleading Justification under the Politics of Terrorism in Saint-Malo. How can the names of malouins merchants on the lists of emigration of the department of the Ille-et-Vilaine be interpreted? Emigration made up of divers persons in exile is a complex concept. The figure of the noble émigré, a stereotype of the counterrevolutionary, is an image created by historiography. Most of the movement of emigration was composed of persons and families from the Third Estate. The malouine society dominated for a long time by a «noble plutocracy of merchant origin» in conflict with a «plebian bourgeoisie» eager for power, was during the Revolution greatly marked by fierce patriotism and was threatened by a monarchical restoration. What, then, are the exact criteria for determining the accusation of emigration leveled against some malouin merchants, some of whom had been anoblis at the end of the seventeenth and at the beginning of the eighteenth centuries?

INDEX

Mots-clés : émigration, Terreur, négociants, négoce, Saint-Malo, Magon

\section{AUTEUR}

\section{KARINE AUDRAN}

Doctorante, Université de Bretagne Sud, Solito (Lorient), 7, rue Raymond Aron, 56100

Lorient, karine.audran@laposte.net 